\title{
COMPARISON OF DEBRIDEMENT EFFICACY AND PERIAPIACL REPAIR USING APICAL NEGATIVE PRESSURE IRRIGATION VERSUS SYRINGE IRRIGATION- A SYSTEMATIC REVIEW
}

\author{
Faisal Alghamdi * and Ahmad H. Almehmadi **
}

\begin{abstract}
Irrigation is considered an indispensable part of root canal treatment as it fortifies the cleaning and disinfection of areas of the root canal system that have been inadequately influenced by instruments. The aim of this systematic review was to collate published data on the two modes of irrigation i.e., conventional irrigation and apical negative pressure irrigation (ANP) for cleaning and shaping of the canals and to compare their efficacy in debridement and periapical tissue repair. The electronic databases PubMed and Google Scholar were searched in this review using specific inclusion and exclusion criteria. The search was performed in June 2018 and updated in March 2019. Among 1481 studies, five studies satisfied the eligibility criteria and were included in the review to be analysed. These studies compared the effectiveness of ANP method against syringe irrigation in removing debris within the root canal system and their effect in periapical tissue repair, including: different types of irrigation methods, debris removal during cleaning and shaping, and updating studies of their effect in periodontal and periapical repair. The compiled data observed that ANP was more efficient in removing the debris and offered a greater advantage that should lead to wider acceptance among dentists for effective results compared to different irrigation systems. However, more clinical trials with standardized protocol and defined clinical, radiographic, and histopathological outcomes with longer follow-up periods are warranted.
\end{abstract}

KEYWORDS: Apical negative pressure irrigation; Conventional irrigation; Syringe irrigation; Debridement; Periapical healing; Periapical Repair

\section{INTRODUCTION}

The most crucial step in root canal treatment is the debridement and removal of necrotic pulp tissue from the root canals of teeth. The complex root canal morphology poses a clinical challenge to achieve complete mechanical debridement ${ }^{(1)}$. It has been observed that about $35 \%$ of the root canal system remains inaccessible for mechanical instrumentation $^{(2)}$. The ideal mode of action of root canal irrigants is to flush out debris, antibacterial and destruction of bacterial by-products, dissolve organic material, and removal of smear layer ${ }^{(3,4)}$. The irrigants must reach the apical region of the root canal in order to remove the bacterial

\footnotetext{
* King Abdulaziz University, Faculty of Dentistry, Department of Oral Biology, Jeddah, Saudi Arabia. ** Assistant Professor, Department of Oral Biology, King Abdulaziz University, Jeddah, KSA.
} 
accumulation, the persistence of which can result in the development of apical periodontitis ${ }^{(5)}$.

The conventional method of irrigation commonly used by general practitioners and endodontists is using syringe and irrigant solution ${ }^{(6,7)}$. The syringe needle is embedded close to the working length (WL) and the irrigant solution is conveyed by which it streams through the canal orifice ${ }^{(8)}$. It is also known as positive pressure irrigation as it creates a pocket of pressure in the apical third of the root canal. Sodium hypochlorite is the most commonly employed root canal irrigant in dental practice and this method of irrigation has been associated with extrusion spills or 'Hypochlorite accidents' ${ }^{(9)}$. Even though it has been widely employed, the major drawback is that it cannot efficiently debride and clean areas other than the main root canal ${ }^{(10,11)}$.

The apical negative pressure irrigation systems (ANP) such as EndoVac ${ }^{\circledR}$ (Discuss Dental, Culver City, ca, Safety Irrigator (Vista Dental, Racine, WI) have been introduced to prevent irrigant extrusion and accelerate the apical irrigation (12). The microcannula of EndoVac ${ }^{\circledR}$ can be inserted till the WL of the root canal, and the generated negative pressure can create a circulation of the irrigant without apical extrusion. The Safety Irrigator features a large coronal evacuation tube that facilitates irrigant aspiration along with simultaneous delivery of the irrigant solution to the root canals through a needle tip. The VPro tip (Vista Dental) produces continuous ultrasonic irrigation using a flexible, 30 -gauge irrigation tip. Some studies have shown that there is better debridement efficacy when compared to positive pressure irrigation ${ }^{(12,13,14)}$.

The primary objective of endodontic therapy in cases of teeth with pulpal necrosis and apical periodontitis is the complete elimination of the micro-organisms along with their by-products from the root canal system. There are multitude of factors that can affect the periapical healing process of endodontically-treated tooth and they include: irrigant solution, irrigation strategies, and intracanal medicaments ${ }^{(15)}$. The control of infection in the root canal system is the most pivotal step in the revitalization process, which in turn leads to the repair of periapical area and continued root development ${ }^{(16)}$. Therefore, the aim of the present systematic review was to systematically collect and analyze the published data on the two different irrigation strategies and their effectiveness in debridement efficacy and periapical tissue repair.

\section{MATERIALS AND METHODS}

This review has been compiled according to the Preferred Reporting Items for Systematic Reviews and Meta-analyses (PRISMA) guidelines.

\section{Research Question}

The following was the research question for the systematic review:

Population: Adult patients with permanent teeth indicated for root canal treatment

Intervention: Irrigation by ANP

Comparison: Conventional syringe irrigation

Outcomes: Removal of debris from root canal system, Periapical Repair

\section{Literature Search}

With respect to the question of the study, we searched the literature and identified relevant studies. The literature search was formulated in June 2018 and then updated in March 2019. The databases searched were both PubMed and Google Scholar. The keywords for our search strategy were "Irrigation", "Negative Pressure" OR "EndoVac", "Syringe" OR "Positive Pressure", "Debris", "Root Canal System", "Apical Periodontitis" OR "Periapical Repair". Using Google Scholar, these terms were entered in these combinations; the terms "Irrigation" were combined with "Negative Pressure" OR "EndoVac", "Syringe" OR "Positive Pressure", the terms "Debris", "Root Canal System", and the terms "Apical Periodontitis" OR "Periapical Repair". When performing PubMed search, the 
keywords were transformed into Medical Subject Heading (MeSH) terms. The MeSH 2018 Browser in the online portal of the U.S National Library of Medicine was utilized to generate $\mathrm{MeSH}$ equivalents wherein "Irrigation", "Negative Pressure", "Syringe", "Dentin Debris", "Root Canal System", "Periapical tissue", "Immature Teeth" and "Periodontitis" were retained in the search. The filters were not applied when combining these terms for the PubMed search in order to retrieve maximum search results. The search database was examined by two reviewers and the final decision for inclusion/exclusion was made according to the following criteria.

\section{Inclusion Criteria}

- Published studies between the 10-year period (2008-2018)

- Original research articles in English language

- Studies performed on humans and animals

\section{Exclusion Criteria}

- Published studies that assessed irrigation systems other than ANP or conventional needle irrigation

- Studies that discuss the irrigation techniques but excluded their effect on apical periodontitis and periapical repair after root canal treatment.

- Review articles on irrigation techniques

\section{Critical Appraisal}

Eligible studies were independently analyzed by the two reviewers according to the eligibility criteria as well as PRISMA guidelines. Any disagreement between the reviewers were resolved using discussion.

\section{Data Extraction and Presentation}

The search strategy using the keywords and MeSH of the databases like PUBMED and Google Scholar yielded a total of 1481 studies, of which
1287 were either unrelated or duplicate topics. Among the potential 194 studies, the eligibility criteria were applied and five studies were included in this systematic review. The summary of the search flow chart for this systematic review has been depicted in [Figure 1].

\section{RESULTS}

The search culminated in five studies that fulfilled both the inclusion and exclusion criteria and they compared the effectiveness of ANP irrigation against syringe irrigation in removing debris within the root canal system and their effect in periapical tissue repair. The outcomes include different types of irrigation methods, debris removal during cleaning and shaping, and updating studies of their effect in the periapical periodontitis and periapical repair. The studies included in this systematic review were five animal studies (in-vivo and invitro) ${ }^{(17-21)}$. With respect to ANP in comparison to syringe irrigation performed, two studies discussed the apical negative pressure and compared it with apical passive ultrasonic and syringe irrigations ${ }^{(17}$, 20). Among the included studies, two of them used a combination of the apical negative pressure and syringe irrigations only, and one study discussed apical negative pressure with syringe irrigations plus the tri-antibiotic intracanal dressing ${ }^{(18,19,21)}$. The included studies showed that there was significant effect in the cleaning efficiency between syringe irrigation and ANP compared with other irrigation systems with reduction in the bacterial load and with respect to periapical repair, it was found that the ANP irrigation gave better biological results and more advanced repair process in immature teeth with apical periodontitis than syringe irrigation (19). In another study, it was found that ANP irrigation presented with mild inflammatory infiltrate, suggestive of an advantage over syringe irrigation for clinical use (20). [Table 1] provides a summary of the included studies in the systematic review. 


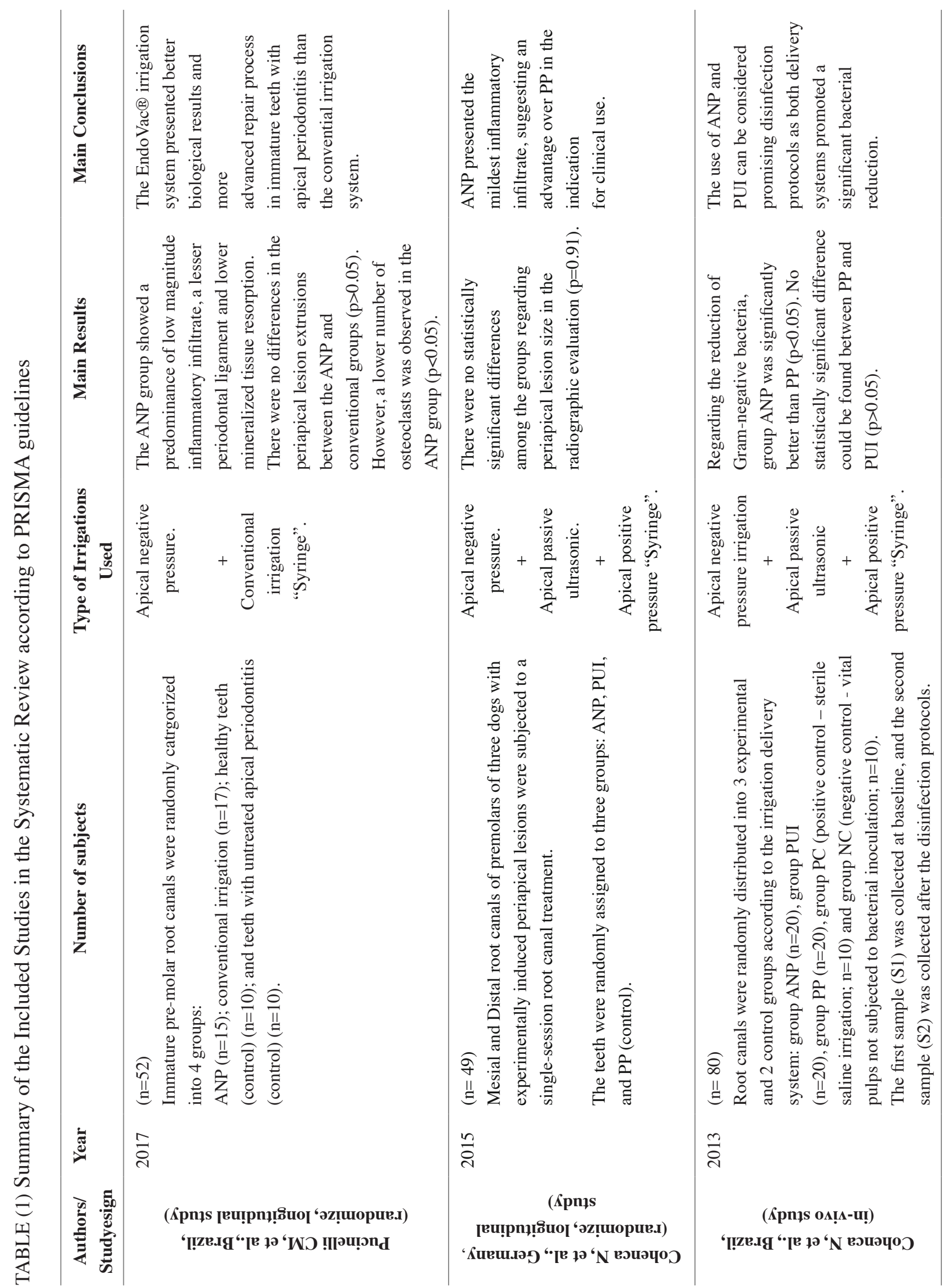




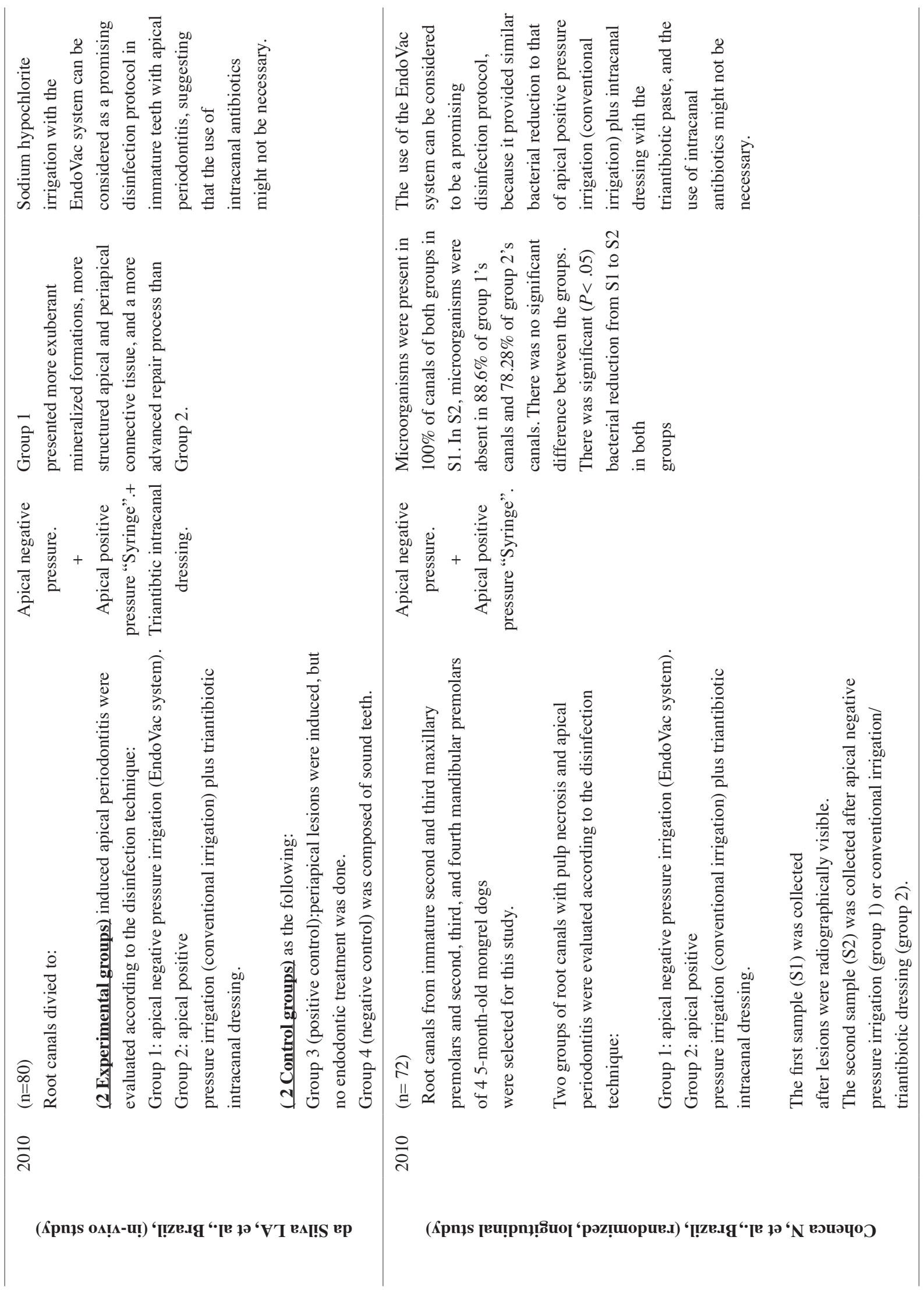




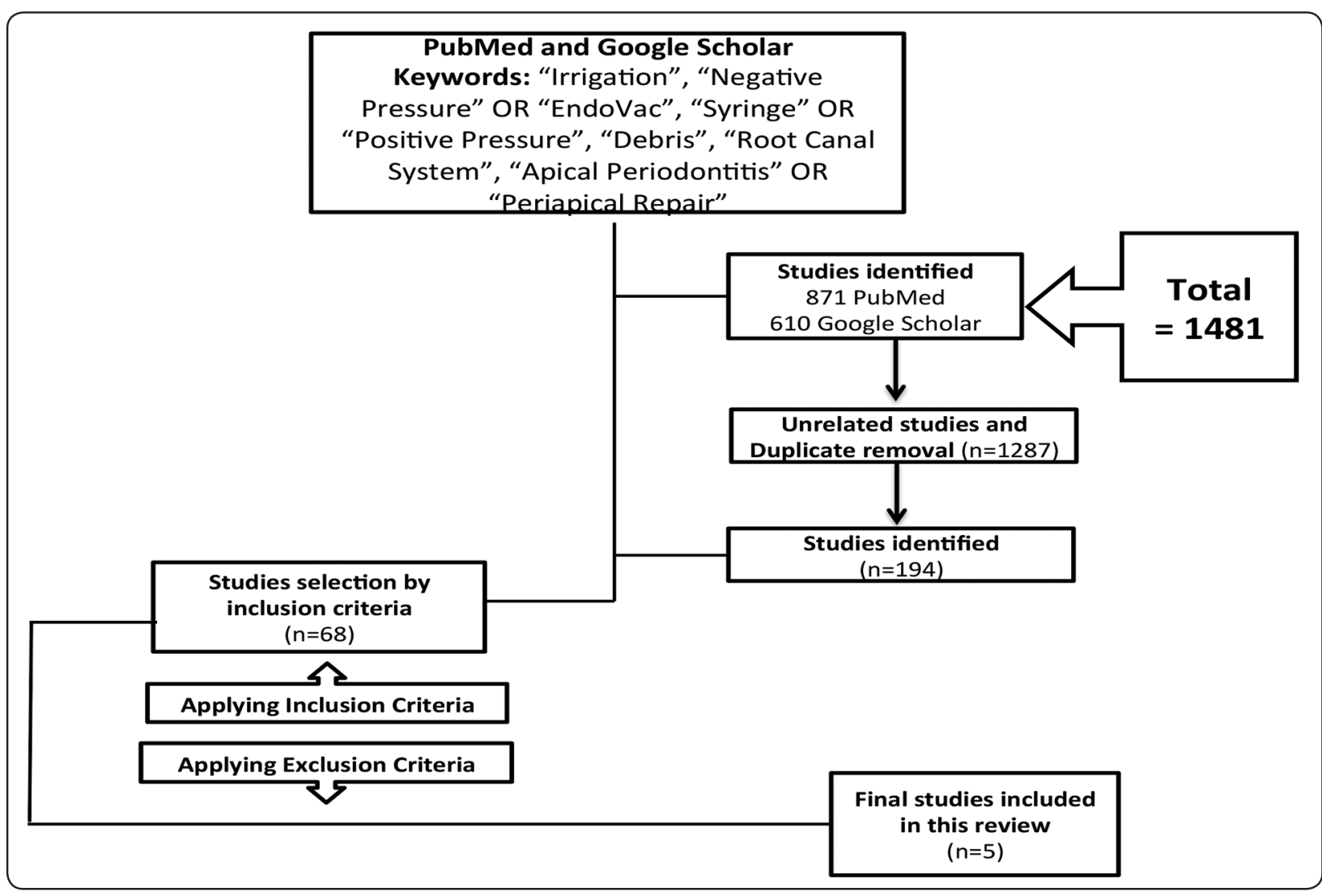

Fig. (1) Flowchart outlining the protocol adopted in the systematic review based on the Preferred Reporting items for Systematic Reviews and Meta-Analyses (PRISMA) guidelines

\section{DISCUSSION}

This systematic review was compiled to assess the efficacy of ANP irrigation when compared to conventional syringe irrigation for outcomes such as removal of debris from the root canal system as well as periapical healing. The outcomes were assessed in five included studies of this review and all of which were performed on dogs' teeth. Although both the outcomes were not assessed in the included studies, the animal studies that compared the ANP and positive pressure irrigation observed that ANP presented with mildest inflammatory infiltrate, the radiographic evaluation showed no significant differences in the size of the periapical lesion and a significant reduction in the microbial load.

The predictors of clinical healing in apical periodontitis can be determined by the outcomes of this systematic review with most significant factor being the assessment of the microbial load. It is to be duly noted that microbes are the key source of periapical pathologies and their persistence can be the major reason for endodontic treatment failures ${ }^{(22,}$ ${ }^{23,24)}$. Infection control is an important component of endodontic therapy as the primary step of reducing the microbial burden is mandatory for initiation of periapical repair. Cohenca et al performed a study on 5-month old mongrel dogs, where the root canals were divided to undergo either ANP or syringe irrigation. It was found that the ANP group had eliminated microbes in $88.6 \%$ of the canals when compared to conventional irrigation ${ }^{(21)}$. A similar study by the same author assessed the difference in the reductions in both Gram-positive and Gramnegative bacteria and it was observed that ANP showed better results when compared to positive 
pressure irrigation ${ }^{(17)}$. But, the drawback in these studies maybe the microbial quantification method using paper-point sampling that has been widely criticized for not being representative of the true microbial load ${ }^{(25)}$.

In the third animal study performed by Cohenca et al, it aimed to assess the periapical repair employing both the irrigation methods using radiographic evaluation and histoenzymology methods. There was no significant difference among both the irrigation methods in the periapical lesion size that was measured after 180 days of root canal treatment, whereas, histopathological results revealed that ANP presented with the mildest inflammatory infiltrate. It was also shown that there was no significant difference in mineralized tissue resorption, periodontal ligament space, and number of osteoclasts ${ }^{(20)}$. This study was unique in assessing the histopathological parameters which is considered as the 'gold standard' for evaluating periapical repair in terms of presence of inflammation and bone resorption ${ }^{(26,27)}$. But these results cannot be extrapolated, as there is a scarcity of comparative studies assessing similar outcomes in root canal treatment.

The studies conducted on dogs' teeth by Pucinelli et al and da Silva et al primarily assessed the histopathological parameters following both the irrigation methods to evaluate the periapical repair and healing. Da Silva et al noted that with ANP irrigation, there was exuberant mineralized tissue formation, structured apical and periapical connective tissue formation, and advanced reparative process when compared to the conventional syringe irrigation ${ }^{(18)}$. Pucinelli et al added that there was higher mineralized tissue formation in the apical region, but there were significant periapical regions which did not undergo substantial repair in the ANP group ${ }^{(19)}$. But the results from these studies state that ANP technique promoted conditions that were favourable for periapical repair due to adequate cleaning and disinfection of the root canal.
It is fundamental that chemicomechanical preparation must lead to successful elimination or reduction in intracanal microbial load that is compatible to periapical healing. Although, the results obtained from the included studies in this review favour ANP irrigation over positive pressure irrigation in terms of microbial, radiologic, and histopathological improvement, the level of evidence remains to be low since they are animal studies. This warrants future randomized controlled clinical trials that scrutinizes each irrigation method with long-term outcome of root canal therapy and its clinical success.

\section{CONCLUSION}

This systematic review concluded that ANP showed better results in certain parameters such as reduction in bacterial load, mild inflammatory infiltrate and improved periapical repair when compared to conventional syringe irrigation. But, there was considerable heterogeneity among the included studies, so inconclusive results suggestive of lack of evidence towards the superiority of a particular irrigation method.

\section{ACKNOWLEDGEMENT}

We thank Prof. Hisham Othman for his review of the paper manuscript, and both Esraa Aljahdali and Reem Alkhanbashi for their help in searching the literature.

\section{REFERENCES}

1. Giardino L, Ambu E, Becce C, Rimondini L, Morra M. Surface tension comparison of four common root canal irrigants and two new irrigants containing antibiotic. J Endod. 2006 Nov;32(11):1091-3.

2. Mitchell RP, Yang S-E, Baumgartner JC. Comparison of apical extrusion of $\mathrm{NaOCl}$ using the EndoVac or needle irrigation of root canals. J Endod. 2010 Feb;36(2):338-41.

3. Gulabivala K, Patel B, Evans G, Ng Y. Effects of mechanical and chemical procedures on root canal surfaces. Endodontic Topics. 2005;10(1):103-22. 
4. Zehnder M. Root canal irrigants. J Endod. 2006 May; 32(5):389-98.

5. Nair PNR. Pathogenesis of apical periodontitis and the causes of endodontic failures. Crit Rev Oral Biol Med Off Publ Am Assoc Oral Biol. 2004 Nov 1;15(6):348-81.

6. Dutner J, Mines P, Anderson A. Irrigation trends among American Association of Endodontists members: a webbased survey. Journal of Endodontics. 2012;38(1):37-40.

7. Willershausen I, Wolf TG, Schmidtmann I, Berger C, Ehlers $\mathrm{V}$, Willershausen B, et al. Survey of root canal irrigating solutions used in dental practices within Germany. Int Endod J. 2015 Jul;48(7):654-60.

8. Boutsioukis C, Van der Sluis L. Syringe irrigation: blending endodontics and fluid dynamics. InEndodontic Irrigation. Springer, Cham; 2015. 45-64 p.

9. HÜlsmann M, RÖdig T. Complications during root canal irrigation. Endodontic Topics. 2007;16(1):27-63.

10. Paqué F, Boessler C, Zehnder M. Accumulated hard tissue debris levels in mesial roots of mandibular molars after sequential irrigation steps: Debris reduction. Int Endod J. 2011 Feb;44(2):148-53.

11. Jiang L-M, Lak B, Eijsvogels LM, Wesselink P, van der Sluis LWM. Comparison of the Cleaning Efficacy of Different Final Irrigation Techniques. J Endod. 2012 Jun;38(6):838-41.

12. Nielsen BA, Craig Baumgartner J. Comparison of the EndoVac system to needle irrigation of root canals. J Endod. 2007 May;33(5):611-5.

13. Shin S-J, Kim H-K, Jung I-Y, Lee C-Y, Lee S-J, Kim E. Comparison of the cleaning efficacy of a new apical negative pressure irrigating system with conventional irrigation needles in the root canals. Oral Surg Oral Med Oral Pathol Oral Radiol Endod. 2010 Mar;109(3):479-84.

14. Siu C, Baumgartner JC. Comparison of the debridement efficacy of the EndoVac irrigation system and conventional needle root canal irrigation in vivo. J Endod. 2010 Nov;36(11):1782-5.

15. Holland R, Gomes Filho JE, Cintra LTA, Queiroz ÍO de A, Estrela C. Factors affecting the periapical healing process of endodontically treated teeth. J Appl Oral Sci. 2017 Oct;25(5):465-76.

16. Tawfik H, Abu-Seida AM, Hashem AA, Nagy MM. Regenerative potential following revascularization of immature permanent teeth with necrotic pulps. Int Endod J. 2013 Oct;46(10):910-22.

17. Cohenca N, Silva L, Silva R, Nelson-Filho P, Heilborn C, Watanabe E, et al. Microbiological evaluation of different irrigation protocols on root canal disinfection in teeth with apical periodontitis: an in vivo study. Brazilian Dental Journal20416538. 2013;24(5):467-73.

18. da Silva LAB, Nelson-Filho P, da Silva RAB, Flores DSH, Heilborn C, Johnson JD, et al. Revascularization and periapical repair after endodontic treatment using apical negative pressure irrigation versus conventional irrigation plus triantibiotic intracanal dressing in dogs' teeth with apical periodontitis. Oral Surg Oral Med Oral Pathol Oral Radiol Endod. 2010 May;109(5):779-87.

19. Pucinelli CM, Silva LAB da, Cohenca N, Romualdo PC, Silva RAB da, Consolaro A, et al. Apical Negative Pressure irrigation presents tissue compatibility in immature teeth. J Appl Oral Sci Rev FOB. 2017 Dec;25(6):612-9.

20. Cohenca N, Romualdo PC, da Silva LAB, da Silva RAB, de Queiroz AM, De Rossi A, et al. Tissue response to root canal irrigation systems in dogs' teeth with apical periodontitis. Clin Oral Investig. 2015 Jun;19(5):1147-56.

21. Cohenca N, Heilborn C, Johnson JD, Flores DSH, Ito IY, da Silva LAB. Apical negative pressure irrigation versus conventional irrigation plus triantibiotic intracanal dressing on root canal disinfection in dog teeth. Oral Surg Oral Med Oral Pathol Oral Radiol Endod. 2010 Jan;109(1):e42-46.

22. Kakehashi S, Stanley Hr, Fitzgerald Rj. The Effects of Surgical Exposures of Dental Pulps In Germ-Free And Conventional Laboratory Rats. Oral Surg Oral Med Oral Pathol. 1965 Sep;20:340-9.

23. Möller AJ, Fabricius L, Dahlén G, Ohman AE, Heyden G. Influence on periapical tissues of indigenous oral bacteria and necrotic pulp tissue in monkeys. Scand J Dent Res. 1981 Dec;89(6):475-84.

24. Siqueira JF. Aetiology of root canal treatment failure: why well-treated teeth can fail. Int Endod J. 2001 Jan;34(1):1-10.

25. Sathorn C, Parashos P, Messer HH. How useful is root canal culturing in predicting treatment outcome? J Endod. 2007 Mar;33(3):220-5.

26. de Paula-Silva FWG, Santamaria M, Leonardo MR, Consolaro A, da Silva LAB. Cone-beam computerized tomographic, radiographic, and histologic evaluation of periapical repair in dogs' post-endodontic treatment. Oral Surg Oral Med Oral Pathol Oral Radiol Endod. 2009 Nov;108(5):796-805.

27. Teixeira RC, Rubira CMF, Assis GF, Lauris JRP, Cestari TM, Rubira-Bullen IRF. Radiological and histopathological evaluation of experimentally-induced periapical lesion in rats. J Appl Oral Sci Rev FOB. 2011 Oct;19(5):500-4. 